\title{
The effect of Silver Diamine Fluoride in Preventing In Vitro Primary Coronal Caries under pH-cycling Conditions
}

\author{
Authors: Parand Sorkhdini ${ }^{\mathrm{a}^{*}}$, Yasmi, O. Crystal ${ }^{\mathrm{b}}$, Qing Tang ${ }^{\mathrm{c}}$, Frank Lippert ${ }^{\mathrm{a}}$ \\ ${ }^{a}$ Department of Cariology, Operative Dentistry and Dental Public Health, Indiana University \\ School of Dentistry, Indianapolis, IN, USA; e-mail: Parsorkh@iu.edu \\ b Department of Pediatric Dentistry, NYU College of Dentistry; New York, USA; e-mail: \\ Yoc1@nyu.edu \\ ${ }^{c}$ Department of Biostatistics, Indiana University School of Medicine, Indianapolis, IN, USA; e- \\ mail: qingtang@iu.edu
}

*Corresponding author:

Parand Sorkhdini

Indiana University School of Dentistry

Department of Cariology, Operative Dentistry and Dental Public Health

Oral Health Research Institute

415 Lansing Street, Indianapolis, IN 46202 (USA)

Tel. +1 317274 3983, Fax +1 317274 5425, E-mail: Parsorkh@iu.edu

This is the author's manuscript of the article published in final edited form as:

Sorkhdini, P., Crystal, Y. O., Tang, Q., \& Lippert, F. (2021). The effect of silver diamine fluoride in preventing in vitro primary coronal caries under pH-cycling conditions. Archives of Oral Biology, 121, 104950. https://doi.org/10.1016/j.archoralbio.2020.104950 


\section{Abstract}

Objectives: This study investigated the ability of SDF, and its individual components, silver $\left(\mathrm{Ag}^{+}\right)$ and fluoride $\left(\mathrm{F}^{-}\right)$ions, in preventing enamel demineralization under $\mathrm{pH}$-cycling conditions in the presence or absence of twice-daily fluoride application.

Design:Polished human enamel specimens were assigned to five treatment groups $(\mathrm{n}=36$ per group): SDF (38\%); SDF followed by application of a saturated solution of potassium iodide $(\mathrm{SDF}+\mathrm{KI})$; silver nitrate $\left(\mathrm{AgNO}_{3}\right.$; silver control, 253,900 ppm Ag); potassium fluoride (KF; fluoride control, 44,800 ppm F); deionized water. Treatments were applied once. Specimens in each treatment group were divided into two subgroups $(n=18)$. During the subsequent 7 -day $\mathrm{pH}-$ cycling phase, specimens were treated twice daily with either 275 ppm fluoride as sodium fluoride or deionized water, immediately before and after a 3-h cariogenic challenge with exposure to artificial saliva at all other times. Changes in color, Vickers surface microhardness (SMH), transverse microradiography (TMR) was calculated. Data were analyzed using two-way ANOVA. Results: In both models, SDF, SDF+KI and KF were superior in inhibiting demineralization compared to $\mathrm{AgNO}_{3}$ and deionized water $(\mathrm{p}<0.0001)$. There was no statistically significant difference between SDF, SDF+KI and KF with twice daily fluoride treatments ( $>0.8)$. However, KF was more effective in preventing demineralization than SDF and SDF+KI in the absence of fluoride treatments $(p=0.0002)$. KI did not affect the ability of SDF to prevent demineralization $(\mathrm{p}>0.4)$.

Conclusion: SDF and SDF+KI appears to be an effective option in preventing primary coronal caries.

Keywords: Silver Diamine Fluoride, Dark staining, Demineralization, pH-cycling, Caries Prevention, Fluoride

\section{Introduction}


Dental caries continues to be one of the most prevalent preventable diseases in the US and worldwide (Kassebaum et al., 2017), with most cases occurring among populations with lowincome and ethnic/racial minority backgrounds (Dye, Thornton-Evans, Li, \& Iafolla, 2015). Current methods of prevention like fluoride varnish applications and sealants, although effective to a large extent, are difficult and costly to implement on a large scale basis in populations who need them the most (Griffin, Wei, Gooch, Weno, \& Espinoza, 2016).

Silver diamine fluoride (SDF) has been rapidly adopted as an agent for caries arrest in dentin caries lesions (Crystal, Janal, Yim, \& Nelson, 2020). Its use as an agent for caries prevention has been studied to a lesser extent, and it is described as a simple and low-cost method that does not require the complex training of the health professional or the cooperation of the patient. This approach may be of great utility as an alternative to more costly preventive methods in communities with limited resources (Llodra et al., 2005).

Remineralization of dental caries lesions and prevention of demineralization at the earliest phase have gained recognition in the minimally invasive approach to dental caries treatment in recent years (Dorri et al., 2017; Sherry Shiqian Gao et al., 2018; Zhi, Lo, \& Lin, 2012). However, current methods of early preventive care are often insufficient to prevent new caries lesions in high risk individuals, (Featherstone \& Doméjean, 2012; Featherstone, Fontana, \& Wolff, 2018) which suggests the need for innovative and alternative preventative approaches for the management and prevention of dental caries that are minimally invasive and less traumatic in children (Selwitz, Ismail, \& Pitts, 2007).

$\mathrm{SDF}$ is a topical solution comprised of silver, ammonia and fluoride $\left(\mathrm{Ag}\left(\mathrm{NH}_{3}\right)_{2} \mathrm{~F}\right)$. It is a safe, effective, efficient, noninvasive and cost-effective method in caries management (Timms, Sumner, Deery, \& Rogers, 2020). The antibacterial properties of silver in addition to the remineralization promotion ability of fluoride act together to both prevent the progression and to arrest dental caries lesions(Mei, Lo, \& Chu, 2018; Timms et al., 2020). Several randomized clinical trials support its use primarily for the treatment of dentin caries, with few studies highlighting its potential usefulness in preventing caries lesion formation (Chu, Lo, \& Lin, 2002; S. S. Gao et al., 2016; Llodra et al., 2005) In addition, there is currently no clinical or in-vitro evidence supporting the use of SDF as a preventative agent on sound enamel. The most significant adverse effect of SDF is non-medical and is the permanent dark staining of the lesion where SDF is applied. This is a major challenge (depending on the visibility of the caries lesions) 
in the acceptance of SDF as a treatment option for some patients/parents (Crystal, Kreider, \& Raveis, 2019; Karched, Ali, \& Ngo, 2019).

Potassium iodide $(\mathrm{KI})$ has been reported and recommended to reduce SDF's dark staining by reacting with free silver ions to form a yellow precipitate of silver iodide (Knight, McIntyre, \& Mulyani, 2006). Nevertheless, there is conflicting evidence about the effectiveness of KI in preventing dark staining without impacting the prevention and arrest of dental caries (Seifo et al., 2020; Sorkhdini, Gregory, Crystal, Tang, \& Lippert, 2020).

pH cycling models are rapid, repeatable, cost effective, and have a higher level of scientific control, and sensitivity to response variables compared to clinical models which makes them ideal to test and evaluate the efficacy of new products (Buzalaf et al., 2010; Lobo, Goncalves, Ambrosano, \& Pimenta, 2005). In addition, $\mathrm{pH}$-cycling models have been validated to evaluate the dose-response effect of fluoride on enamel and subclinical enamel carious lesions (Featherstone, Stookey, Kaminski, \& Faller, 2011). For these reasons, the $\mathrm{pH}$-cycling model based on that described by Featherstone et al. (2011) (Featherstone et al., 2011) were used in this study to determine, 1) the efficacy of SDF in preventing enamel caries lesion formation under $\mathrm{pH}$ cycling conditions in the presence or absence of twice-daily fluoride or placebo treatments, and 2) to evaluate staining and caries prevention of $\mathrm{SDF}+\mathrm{KI}$.

We hypothesized that a) SDF is still effective in enamel caries prevention with twice-daily fluoride application, and b) applying KI after SDF application can mitigate dark staining and at the same time does not negatively affect the anti-caries ability of SDF.

\section{Materials and Methods}

\subsection{Study Design}

The study was exempted from Institutional Review Board (IRB) supervision IRB \#: NS0911-07. The flow chart of the study is shown in Fig. 1. One hundred and eighty polished human permanent enamel samples were allocated to five treatment groups after color and surface microhardness assessments: SDF, SDF+ Potassium iodide (KI), Silver nitrate $\left(\mathrm{AgNO}_{3}\right)$, Potassium fluoride $(\mathrm{KF})$, and deionized water. The study performed color assessment immediately after the treatment application. Enamel samples were then randomized into two $\mathrm{pH}$-cycling groups: 1- $\mathrm{pH}$-cycling 
with fluoride intervention and 2- $\mathrm{pH}$-cycling with placebo. Specimens were $\mathrm{pH}$ cycled for 7 days. All specimens were then evaluated for changes in color using a colorimeter, Vickers surface microhardness and utilizing transverse microradiography to measure integrated mineral loss and lesion depth.

\subsection{Specimen selection and preparation}

This experiment used one hundred and eighty sound extracted human permanent teeth (buccal and/or lingual surfaces of predominantly molars and premolars from dental clinics' anonymous donations were used) with no wear, cracks, or defects. Each tooth was cut into $4 \times 4 \mathrm{~mm}$ specimens, then ground and polished to create flat specimens, as previously descried (Sorkhdini et al., 2020). Nikon SMZ 1500 stereomicroscope at 20× magnification was used to check the enamel samples with a thickness range of 1.7-2.2 mm for flaws. The percentage of specimen losses during preparation was $30 \%$, and no specimen was lost during the experimental procedure. The final samples were kept at $100 \%$ relative humidity at $4^{\circ} \mathrm{C}$.

\subsection{Pretreatment assessment}

\subsubsection{Sound enamel color assessment}

Changes in color were measured using a spectrophotometer, as described previously, using Minolta Chroma meter CR-241 (Minolta Camera Co., Osaka, Japan) with D65 light against a white background (Alshara, Lippert, Eckert, \& Hara, 2014). Commision Internationale de l'Eclairage (CIE) L* values were recorded, and all measurements were repeated three times.

\subsubsection{Sound enamel surface microhardness (SMH)}

Specimens were evaluated for sound enamel surface microhardness utilizing a microhardness tester as described previously (2100 HT; Wilson Instruments, Norwood, MA, USA) (Sorkhdini et al., 2020). Image analysis software (Clemex CMT HD version 6.0.011, Clemex Technologies Inc., Longueuil, Quebec, Canada) was used to determine the indentation length of the SMHsound. Enamel samples which fulfilled the criteria of $300 \leq$ SMHsound $\leq 400$ were used in this study.

\subsubsection{Specimen stratification}


Between treatment and study groups, one hundred and eighty enamel specimens were stratified into five treatment groups with 36 specimens per treatment group to ensure that there were no significant differences in SMHsound.

\subsection{Treatment groups}

Enamel samples were randomized into five treatment groups of 36 samples each: SDF, SDF+KI, $\mathrm{AgNO}_{3}, \mathrm{KF}$ and deionized water (placebo).

- $\quad$ SDF: 38\% SDF (Advantage Arrest, Elevate Oral Care LLC, Florida, USA) solution; nominally 253,900 ppm Ag; 44,800 ppm F (Advantage Arrest, Fl, USA)

- $\quad \mathrm{SDF}+\mathrm{KI}$ : SDF application followed by supersaturated KI application (Potassium iodide 39\% w/v solution, 30315, Sigma-Aldrich, St. Louis, US)

- $\quad \mathrm{AgNO}_{3}$ : silver control; 253,900 ppm Ag (Silver nitrate 31630, Sigma-Aldrich, St. Louis, USA)

- $\quad$ KF: fluoride control; 44,800 ppm F (Potassium fluoride 60238, Sigma-Aldrich, St. Louis, USA)

- Deionized water: negative control

A micro applicator (Regular; Premium Plus International Ltd., Hong Kong, China) was used to apply SDF solution to the enamel surface. $\mathrm{KI}, \mathrm{KF}, \mathrm{AgNO}_{3}$ and deionized water were applied using a micro-brush (Premium Plus Regular Tip Micro A microbrush). For the SDF+KI group, after SDF application saturated KI solution was applied immediately until the creamy yellow solution turned clear (Zhao, Mei, Burrow, Lo, \& Chu, 2017a). All solutions were left on the enamel surface undisturbed for $60 \mathrm{~min}$.

\subsection{Post-treatment color assessment}

After the 60-minute treatment the reaction products were rubbed off using sterile cotton swabs. Immediately afterwards, change in color was assessed again as described above. $\mathrm{L}^{*}$ was recorded for each sample and $\Delta \mathrm{L}^{*}$ calculated as follows: $\Delta \mathrm{L}^{*}=\mathrm{L}^{*}$ post $-\mathrm{L}^{*}$ sound.

\section{6 pH-cycling}

Immediately after color measurements, 36 enamel specimens from each treatment group were allocated to two intervention groups to ensure no significant differences in SMH sound: 
1- $\mathrm{pH}$-cycling with fluoride intervention model: $\mathrm{pH}$-cycling with fluoride solutions containing 275 ppm fluoride. The fluoride concentration corresponds to the dilution (1:3) of dentifrices containing 1100 ppm $\mathrm{F}$ in the oral cavity during toothbrushing. The fluoridated solution was prepared with $\mathrm{NaF}$ (Sodium fluoride, 97\%, extra pure, 191270250, Acros Organics, New Jersey, USA) and deionized water.

2- pH-cycling with placebo model: deionized water (i.e. fluoride-free placebo) as a control Eighteen blocks from each treatment group were submitted for five days of $\mathrm{pH}$-cycling utilizing a protocol based on that by Featherstone et al. (2011) (Featherstone et al., 2011), followed by two days of storage in a remineralizing solution. The blocks were kept individually in a demineralizing solution (2.0 mM calcium, $2.0 \mathrm{mM}$ phosphate, $0.030 \mathrm{ppm} \mathrm{F}$, in $75 \mathrm{mM}$ Acetic Acid, pH 4.3) for 3 $\mathrm{h}$ and in a remineralizing solution $(1.5 \mathrm{mM}$ calcium, $0.9 \mathrm{mM}$ phosphate, $150 \mathrm{mM}$ of $\mathrm{KCl}, 0.050$ ppm $\mathrm{F}^{-}$in $20 \mathrm{mM}$ cacodylic buffer, $\mathrm{pH} \mathrm{7.4)}$ ) for $21 \mathrm{~h}$. Twice a day (before and after immersion in the demineralizing solution), the blocks were washed with deionized water and subjected to oneminute immersion in fluoride or deionized water treatments. This cycle was repeated for five days and the enamel blocks then remained in the remineralizing solution for two days. All treatments were performed at a ratio of $10 \mathrm{ml}$ of solution per specimen and all solutions were renewed prior to the start of each treatment. All specimens were washed with deionized water before and after each immersion in the solutions. The remineralization and demineralization were carried out in the incubator at $37^{\circ} \mathrm{C}$. After completion of the $\mathrm{pH}$ cycling phase, all specimens were rinsed with deionized water and stored at $100 \%$ relative humidity at $4{ }^{\circ} \mathrm{C}$.

\subsection{Post-intervention assessment}

\subsubsection{Post-intervention color assessment}

Change in color was assessed on all samples after $\mathrm{pH}$ cycling as described above. L* was recorded for each sample and this variable was calculated: $\Delta \mathrm{L}^{*}$ intervention $=\mathrm{L}^{*}{ }_{\text {intervention }}-\mathrm{L}^{*}$ sound. All the color assessment measurements were conducted three times, and the mean was reported.

\subsubsection{Surface microhardness change}

All samples underwent surface microhardness assessment as described above. On the right of the baseline indentations, a second set of four indentations was inserted on each sample, yielding 
SMHpost. The percent change in surface microhardness for each sample was determined as follows: \%SMHchange $=100 \times($ SMHsound - SMHpost $) /$ SMHsound .

\subsection{Transverse Microradiography}

One section per samples, approximately $100 \mu \mathrm{m}$ in thickness, was cut from the center of each sample and across the lesion window and sound enamel areas using a Silverstone-Taylor Hard Tissue Microtome (Scientific Fabrications Laboratories, USA) as described previously (Sorkhdini et al., 2020). $\Delta \mathrm{Z}$ - integrated mineral loss (product of lesion depth and the mineral loss over that depth), L - lesion depth were documented for each sample section.

\section{$2.9 \quad$ Statistical Analysis}

The study had $80 \%$ power to detect a difference of $10 \%$ for $\% \mathrm{SMHchange,} 15 \%$ for $\Delta \mathrm{Z}, 27 \%$ for L and $15 \%$ for color changes $\left(\Delta \mathrm{L}^{*}\right)$, with an overall sample size of 18 samples per group. The calculations assumed two-sided tests performed at a 5\% significance level for each type of comparison, with coefficients of variance estimated at 0.1 for $\% \mathrm{SMHchange,} 0.15$ for $\Delta \mathrm{Z}, 0.27$ for $\mathrm{L}$ and 0.15 for color changes $\left(\Delta \mathrm{L}^{*}\right) . \% \mathrm{SMHchange}, \Delta \mathrm{Z}, \mathrm{L}$, and color changes were evaluated using two-way ANOVA to assess the efficacy of different types of treatments and models, as well as interactions between treatment types and models. All pair-wise comparisons from ANOVA analysis were produced using Fisher's Protected Least Significant Differences to control the overall significance level at 5\%. Analyses were completed using SAS version 9.4 (SAS Institute, Inc., Cary, NC).

\section{Results}

\subsection{Microhardness}

The two-way interaction between treatment types and models was significant $(p=0.01)$. The percent change in surface microhardness (\%SMHchange) data for both $\mathrm{pH}$-cycling with fluoride intervention and placebo model are shown in Fig. 2.

In the $\mathrm{pH}$-cycling with fluoride model there were no statistically significant differences between $\mathrm{SDF}$ and SDF+KI in preventing enamel demineralization $(\mathrm{p}=0.992)$. There were no statistically significant differences between $\mathrm{SDF}, \mathrm{SDF}+\mathrm{KI}$ and $\mathrm{KF}(\mathrm{p}>0.8)$ and they were all more effective in 
preventing demineralization than $\mathrm{AgNO}_{3}$ and deionized water $(\mathrm{p}<0.0001)$. There was no difference between $\mathrm{AgNO}_{3}$ and deionized water $(\mathrm{p}=0.91)$.

In the $\mathrm{pH}$-cycling with placebo model there were no statistically significant differences between $\mathrm{SDF}$ and $\mathrm{SDF}+\mathrm{KI}$ in preventing enamel demineralization $(\mathrm{p}=0.4410)$. However, KF was more effective in preventing caries lesion formation than SDF and $\mathrm{SDF}+\mathrm{KI}(\mathrm{p}<0.00001)$. SDF, $\mathrm{SDF}+\mathrm{KI}$ and KF were more effective in preventing demineralization than $\mathrm{AgNO}_{3}$ and deionized water $(p<0.0001)$. There was no difference between $\mathrm{AgNO}_{3}$ and deionized water $(\mathrm{p}=0.6747)$.

$\mathrm{SDF}, \mathrm{SDF}+\mathrm{KI}, \mathrm{AgNO}_{3}$ and deionized water treatments resulted in significantly less demineralization with twice-daily fluoride applications than with placebo treatments $(\mathrm{p}<0.05)$. In the KF group there was no statistically significant difference between $\mathrm{pH}$-cycling with fluoride and placebo models in preventing enamel demineralization $(p=0.510)$.

\subsection{Transverse Microradiography}

The two-way interaction between treatment types and models was significant $(\mathrm{p}=0.001)$. The $\Delta \mathrm{Z}$ data for both $\mathrm{pH}$-cycling with fluoride and placebo models are shown in Fig. 3. In the pH-cycling with fluoride model there was no difference in $\Delta \mathrm{Z}$ between treatment groups (all $\mathrm{p}>0.15$ ). However, in the $\mathrm{pH}$-cycling with placebo model there were statistically significant differences in $\Delta \mathrm{Z}$ between $\mathrm{SDF}, \mathrm{SDF}+\mathrm{KI}$, and $\mathrm{KF}$ compared to $\mathrm{AgNO}_{3}$ and deionized water (all $\mathrm{p}<0.01$ ).

There were statistically significant differences between $\mathrm{pH}$-cycling with fluoride and placebo models for the $\mathrm{SDF}+\mathrm{KI}, \mathrm{AgNO}_{3}$, and deionized water groups $(\mathrm{p}<0.0001)$. However, the diffrence between $\mathrm{pH}$-cycling with fluoride and placebo models was not significant for the SDF and KF groups (both $\mathrm{p}>0.05$ ).

The two-way interaction between treatment types and models was not significant $(\mathrm{p}=0.096)$; however, both factors were (treatment types $-\mathrm{p}=0.002$; models $-\mathrm{p}=0.001$ ). The L data for both $\mathrm{pH}$-cycling with fluoride and placebo models are shown in Fig. 4. In both pH-cycling with fluoride model there were statistically significant differences between SDF, SDF+KI, and KF compared to $\mathrm{AgNO}_{3}$ and deionized water $(\mathrm{p}<0.0001)$. There were statistically significant differences between $\mathrm{pH}$-cycling with fluoride intervention and placebo models $(\mathrm{p}=0.001)$.

No differences in mean lesion mineral distributions between study groups were noted (data not shown). 


\subsection{Color Assessment}

The two-way interaction between treatment types and models was significant $(\mathrm{p}=0.002)$. The $\Delta \mathrm{L}^{*}$ data for both $\mathrm{pH}$-cycling models and all treatment groups are shown in Fig. 5. Irrespective of the $\mathrm{pH}$ cycling model and considering only color changes after treatment application, $\mathrm{SDF}$ and $\mathrm{AgNO}_{3}$ groups presented significantly lower $\Delta \mathrm{L}^{*}$ values compared to $\mathrm{SDF}+\mathrm{KI}, \mathrm{KF}$ and deionized water between baseline and post-intervention $(\mathrm{p}<0.0001)$. Moreover, in SDF group $\Delta \mathrm{L}^{*} \operatorname{did}$ not significantly change after $\mathrm{pH}$-cycling intervention in both models $(\mathrm{p}<0.0001)$. In both $\mathrm{pH}$-cycling models, $\Delta \mathrm{L}^{*}$ values from baseline to post-intervention shown $\mathrm{SDF}+\mathrm{KI}$ groups had significantly higher $\Delta \mathrm{L}^{*}$ values than SDF alone $(\mathrm{p}<0.0001)$. In the $\mathrm{pH}$-cycling with placebo model, the deionized water group demonstrated statistically significant increase in $\Delta \mathrm{L}^{*}$ compared to all other groups from baseline to post-intervention $(\mathrm{p}<0.0001)$.

Twice-daily fluoride application in the $\mathrm{pH}$-cycling with fluoride intervention did not affect the $\mathrm{L}^{*}$ values compared to $\mathrm{pH}$ cycling with placebo in the $\mathrm{SDF}, \mathrm{SDF}+\mathrm{KI}$ and $\mathrm{KF}$ groups $(\mathrm{p} \geq 0.0535)$. In the $\mathrm{AgNO}_{3}$ and deionized water groups, $\mathrm{pH}$-cycling with placebo significantly increased $\Delta \mathrm{L}^{*}$ values $(\mathrm{p}<0.0001)$.

\section{Discussion}

SDF has gained growing popularity in treating dentin caries. However, there is insufficient evidence about the caries preventive effect of SDF on enamel. To the authors' knowledge, no experimental data about the ability of SDF in preventing enamel demineralization under $\mathrm{pH}$ cycling conditions in the presence or absence of twice-daily fluoride application exists. Therefore, this study is innovative because it is the first to examine the specific effect of SDF and SDF+KI as a caries preventative agent on sound enamel.

The chosen pH-cycling model is based on the model by Featherstone et al. (2011) (Featherstone et al., 2011) which is a net demineralization model (Featherstone et al., 2011). This study mimicked in vivo caries formation in a high-risk patient as a professionally applied intervention was followed by twice-daily at-home application of over-the-counter fluoride toothpaste. This model was able to distinguish between $\mathrm{SDF}, \mathrm{SDF}+\mathrm{KI}$ and $\mathrm{KF}$ vs. $\mathrm{AgNO}_{3}$ and deionized water, highlighting longitudinal effects of the fluoride-containing interventions that persisted even after $\mathrm{pH}$ cycling. 
The model was also sensitive enough to show the effect of twice-daily fluoride application during the $\mathrm{pH}$ cycling phase in addition to aforementioned intervention effects.

The present findings suggest that SDF appears to offer an alternative approach in preventing primary coronal caries. Furthermore, KI application after SDF significantly improved the dark staining without affecting the inhibition of demineralization ability of SDF. These findings are highly consistent and strongly support our study hypotheses.

Based on the findings of this study, SDF and SDF+KI are more effective in inhibiting demineralization and promoting remineralization than $\mathrm{AgNO}_{3}$ and deionized water (Fig. 2). Accordingly, application of KI after SDF did not affect the ability of SDF to prevent demineralization (Fig. 2). These results are in agreement with our previous work using a chemical model to induce demineralization on sound enamel (Sorkhdini et al., 2020) and a biofilm study employing dentin specimens (Knight et al., 2005).

In both models, SDF, SDF+KI and KF were more effective in their ability to prevent demineralization and promote remineralization than $\mathrm{AgNO}_{3}$ and deionized water (Fig. 2). Moreover, there was no difference between SDF, SDF+KI and KF with twice daily fluoride treatments. Nevertheless, KF was superior in preventing demineralization and promoting remineralization than SDF and SDF $+\mathrm{KI}$ in $\mathrm{pH}$-cycling with placebo. These results clearly indicate that the caries preventive effect of SDF in this model is a function of the fluoride content and not the silver component or any combination of the two.

Mei et al. mentioned in their review article on dentin that the combination of silver and fluoride in an alkaline solution has a synergistic effect in arresting dentin caries as silver ions inhibit biofilm growth, whereas fluoride enhances mineral formation, which make SDF different from other fluoride agents (Mei et al., 2018). However, the role of silver and fluoride ions on the preventive effect of SDF on sound enamel, is still unclear.

Fluoride, which is the other component of SDF, plays an important role in promoting remineralization and inhibiting enamel demineralization. Fluoride enhances the speed of enamel remineralization and slows down the enamel dissolution process. Fluoride has also been demonstrated to have anti-bacterial activity which may prevent production of acids by bacteria (Hu, Meyer, \& Duggal, 2018; Mei, Zhao, Ito, Lo, \& Chu, 2016). However, it is still not clear to what extent silver and fluoride ions in SDF display their mechanism of action when applied to enamel (Hu et al., 2018). 
Based on the VHN results of both $\mathrm{pH}$-cycling models, KF was equally or more effective than SDF. There are several hypotheses we consider as a rationale:

1) Silver ions in SDF (loosely) bound to the enamel surface may act as a barrier for effective remineralization to occur. It is possible that residual silver ions after SDF application or silver ions released from bound silver compounds solubilized during phases of demineralization, which can reprecipitate again, did impair remineralization. This may have reduced the effectiveness of fluoride initially applied as part of the SDF intervention (Zhi, Lo, \& Kwok, 2013).

2) Secondly, This could be explained by the fact that silver ions seem to precipitate in the pellicle when applied to sound enamel surfaces, which would mean that most of the observed effects are due to the remineralizing action of the higher concentration of fluoride (Li et al., 2019). 3) Thirdly, fluoride ions from SDF may be hampered from entering the sound enamel since there was no lesion to penetrate. As sound enamel has very low porosity and SDF has high reactivity with the enamel surface, the remineralizing effect of fluoride in SDF may have been decreased (Rosenblatt, Stamford, \& Niederman, 2009).

4) Lastly, no cariogenic biofilms were included in the $\mathrm{pH}$-cycling models. Thus, the antibacterial effect of silver was not considered in the present study. The transverse microradiography data (Figs. 3 and 4) largely mirrored the hardness data, although differences between treatment groups and models were less pronounced. This may be due to the lack of sensitivity of the transverse microradiography technique in precisely evaluating the mineral status of very early, shallow lesions, which agrees with a previous study (Sorkhdini et al., 2020).

SDF has been proven to be a relatively safe topical agent from a pharmacokinetics perspective (Vasquez et al., 2012). However, the pronounced and permanent black staining of SDF is a significant aesthetic barrier which greatly impacts its adoption into everyday practice. Parents believe the dark staining on the tooth surface would result in harming psychosocial consequences to their child due to the judgments of other individuals (Crystal, Kreider, et al., 2019; Roberts et al.). Hence, diminishing the black staining caused by SDF would greatly enhance the opportunity for its universal use (Crystal, Kreider, et al., 2019; Garg, Sadr, \& Chan, 2019).

In this study, dark staining was observed on the enamel specimens treated with either SDF or $\mathrm{AgNO}_{3}$ (Fig. 5). These results were in agreement with studies on dentin (Zhi et al., 2013) and enamel (Sorkhdini et al., 2020). The extent of staining caused by SDF did not subside during the 
$\mathrm{pH}$ cycling phase, suggesting that the stain is of a tenacious nature and resists repeated acid challenges.

In both pH-cycling models, application of KI after SDF considerably reduced the discoloration caused by SDF (Fig. 5). This was in agreement with the outcomes of other studies performed on enamel and dentin (Gupta, Thomas, Radhakrishna, Srikant, \& Ginjupalli, 2019; Zhao et al., 2019). Moreover, KI was able to permanently prevent staining in enamel caused by SDF-related in both pH-cycling models (Fig. 5).

As KF group was strong anti caries treatment the $\mathrm{pH}$-cycling did not affect the color of specimen and did not create white opacity lesion due to demineralization. We noticed an interesting result in both $\mathrm{AgNO}_{3}$ groups in that the staining persisted throughout the $\mathrm{pH}$ cycling phase in the fluoride-treated specimens but not in those treated with deionized water (Fig. 5). It is likely that the repeated fluoride application resulted in more persistent binding of silver ions, whereas in the absence of fluoride the daily acid challenge lead to continuous dissolution of enamel-bound silver. However, $\mathrm{AgNO}_{3}$ treatment per se did not affect demineralization as demonstrated using a different model previously (Sorkhdini et al., 2020; Zhao, Mei, Burrow, Lo, \& Chu, 2017b; Zhi et al., 2013). This suggests different reaction products between SDF and enamel vs. $\mathrm{AgNO}_{3}$ and enamel which warrants further exploration. Lastly, in the pH-cycling with placebo model, the deionized water group demonstrated significantly more whiteness than all other groups after $\mathrm{pH}$ cycling intervention because of the formation of white opacity demineralization lesions (Fig. 5).

Several limitations must be considered in the interpretation of the present results. The chosen $\mathrm{pH}$ cycling period was relatively short, and a longer duration may have been useful to predict potentially longitudinal effects of SDF in enamel caries prevention. A longer phase would have also allowed to evaluate the impact of a repeat SDF application as conducted clinically. (Crystal, Rabieh, Janal, Rasamimari, \& Bromage, 2019). Undoubtedly, pH-cycling models have their own limitations. as they only partially replicate the complex clinical conditions of caries dynamics and natural oral environments (Zhao, Mei, Li, Lo, \& Chu, 2017). It is inappropriate to compare it directly with the clinical situation. Similarly, it is difficult to compare with the biofilm models used to simulate cariogenic biofilm effects, as these are more complex than a simple, chemical pHcycling model. The major limitation with regards to chemical $\mathrm{pH}$-cycling is the lack of bacteria and pellicle which are naturally present in the oral environment. For example, acid diffusion is completely altered by the gradient that is caused by an extracellular biofilm matrix, which also 
controls ion exchange between the enamel surface and the external environment. Ultimately, the $\mathrm{pH}$-cycling model is simplistic.

Our results warrant further longitudinal investigation with bacterial $\mathrm{pH}$-cycling models comprising biofilm development to simulate a natural process occurring in the oral cavity to better understand the efficacy of SDF compared to SDF+KI and its individual components.

Under the conditions of this study, SDF appears to be an effective topical agent in the prevention of enamel caries with the mode of action being effective delivery of high concentrations of fluoride ion to the sound enamel surface. While KI helped prevent dark staining caused by SDF, KI did not impair SDF's ability to prevent chemical demineralization. Further clinical research is required to confirm the caries preventive ability of SDF and SDF+KI on enamel.

\section{Acknowledgements}

The authors would like to thank Professor John D.B. Featherstone for his precious comments, insightful suggestions, and careful reading of this manuscript. This work was supported by the Indiana University School of Dentistry Department of Cariology, Operative Dentistry and Dental Public Health and by the Oral Health Research Institute Remineralization Research Program. The authors are indebted to Elevate Oral Care LLC, Florida, USA for providing SDF (Advantage Arrest) free of charge.

\section{References}

Alshara, S., Lippert, F., Eckert, G. J., \& Hara, A. T. (2014). Effectiveness and mode of action of whitening dentifrices on enamel extrinsic stains. Clin Oral Investig, 18(2), 563-569. doi:10.1007/s00784-013-0981-8

Buzalaf, M. A., Hannas, A. R., Magalhaes, A. C., Rios, D., Honorio, H. M., \& Delbem, A. C. (2010). $\mathrm{pH}$-cycling models for in vitro evaluation of the efficacy of fluoridated dentifrices for caries control: strengths and limitations. J Appl Oral Sci, 18(4), 316-334. doi:10.1590/s1678-77572010000400002

Chu, C. H., Lo, E. C., \& Lin, H. C. (2002). Effectiveness of silver diamine fluoride and sodium fluoride varnish in arresting dentin caries in Chinese pre-school children. J Dent Res, 81(11), 767-770. doi:10.1177/0810767 
Crystal, Y. O., Janal, M. N., Yim, S., \& Nelson, T. (2020). Teaching and utllization of SDF and Hall-style crowns in US pediatric dentistry residency programs. $J$ Am Dent Assoc, in press.

Crystal, Y. O., Kreider, B., \& Raveis, V. H. (2019). Parental Expressed Concerns about Silver Diamine Fluoride (SDF) Treatment. J Clin Pediatr Dent, 43(3), 155-160. doi:10.17796/1053-4625-43.3.2

Crystal, Y. O., Rabieh, S., Janal, M. N., Rasamimari, S., \& Bromage, T. G. (2019). Silver and fluoride content and short-term stability of $38 \%$ silver diamine fluoride. J Am Dent Assoc, 150(2), 140-146. doi:10.1016/j.adaj.2018.10.016

Dorri, M., Martinez-Zapata, M. J., Walsh, T., Marinho, V. C., Sheiham Deceased, A., \& Zaror, C. (2017). Atraumatic restorative treatment versus conventional restorative treatment for managing dental caries. The Cochrane Database of Systematic Reviews, 12(12), CD008072-CD008072. doi:10.1002/14651858.CD008072.pub2

Dye, B. A., Thornton-Evans, G., Li, X., \& Iafolla, T. J. (2015). Dental caries and sealant prevalence in children and adolescents in the United States, 2011-2012. NCHS Data Brief(191), 1-8.

Featherstone, J. D., \& Doméjean, S. (2012). The role of remineralizing and anticaries agents in caries management. Adv Dent Res, 24(2), 28-31. doi:10.1177/0022034512452885

Featherstone, J. D., Fontana, M., \& Wolff, M. (2018). Novel Anticaries and Remineralization Agents: Future Research Needs. J Dent Res, 97(2), 125-127. doi:10.1177/0022034517746371

Featherstone, J. D., Stookey, G. K., Kaminski, M. A., \& Faller, R. V. (2011). Recommendation for a non-animal alternative to rat caries testing. Am J Dent, 24(5), 289-294.

Gao, S. S., Zhao, I. S., Duffin, S., Duangthip, D., Lo, E. C. M., \& Chu, C. H. (2018). Revitalising Silver Nitrate for Caries Management. International journal of environmental research and public health, 15(1), 80. doi:10.3390/ijerph15010080

Gao, S. S., Zhao, I. S., Hiraishi, N., Duangthip, D., Mei, M. L., Lo, E. C. M., \& Chu, C. H. (2016). Clinical Trials of Silver Diamine Fluoride in Arresting Caries among Children: A Systematic Review. JDR Clin Trans Res, 1(3), 201-210. doi:10.1177/2380084416661474

Garg, S., Sadr, A., \& Chan, D. (2019). Potassium Iodide Reversal of Silver Diamine Fluoride Staining: A Case Report. Oper Dent, 44(3), 221-226. doi:10.2341/17-266-S

Griffin, S. O., Wei, L., Gooch, B. F., Weno, K., \& Espinoza, L. (2016). Vital Signs: Dental Sealant Use and Untreated Tooth Decay Among U.S. School-Aged Children. MMWR. Morbidity and mortality weekly report, 65(41), 1141-1145. doi:10.15585/mmwr.mm6541e1. (Accession No. 27764075)

Gupta, J., Thomas, M. S., Radhakrishna, M., Srikant, N., \& Ginjupalli, K. (2019). Effect of silver diamine fluoride-potassium iodide and $2 \%$ chlorhexidine gluconate cavity cleansers on the bond strength and microleakage of resin-modified glass ionomer cement. $J$ Conserv Dent, 22(2), 201-206. doi:10.4103/jcd.Jcd_485_18

Hu, S., Meyer, B., \& Duggal, M. (2018). A silver renaissance in dentistry. Eur Arch Paediatr Dent, 19(4), 221-227. doi:10.1007/s40368-018-0363-7

Karched, M., Ali, D., \& Ngo, H. (2019). In vivo antimicrobial activity of silver diammine fluoride on carious lesions in dentin. J Oral Sci, 61(1), 19-24. doi:10.2334/josnusd.170366

Kassebaum, N. J., Smith, A. G. C., Bernabe, E., Fleming, T. D., Reynolds, A. E., Vos, T., . . . Collaborators, G. B. D. O. H. (2017). Global, Regional, and National Prevalence, 
Incidence, and Disability-Adjusted Life Years for Oral Conditions for 195 Countries, 1990-2015: A Systematic Analysis for the Global Burden of Diseases, Injuries, and Risk Factors. J Dent Res, 96(4), 380-387. doi:10.1177/0022034517693566

Knight, G. M., McIntyre, J. M., Craig, G. G., Mulyani, Zilm, P. S., \& Gully, N. J. (2005). An in vitro model to measure the effect of a silver fluoride and potassium iodide treatment on the permeability of demineralized dentine to Streptococcus mutans. Aust Dent J, 50(4), 242-245. doi:10.1111/j.1834-7819.2005.tb00367.x

Knight, G. M., McIntyre, J. M., \& Mulyani. (2006). The effect of silver fluoride and potassium iodide on the bond strength of auto cure glass ionomer cement to dentine. Aust Dent $J$, 5l(1), 42-45. doi:10.1111/j.1834-7819.2006.tb00399.x

Li, Y., Liu, Y., Psoter, W. J., Nguyen, O. M., Bromage, T. G., Walters, M. A., . . Kumararaja, F. C. (2019). Assessment of the Silver Penetration and Distribution in Carious Lesions of Deciduous Teeth Treated with Silver Diamine Fluoride. Caries Res, 53(4), 431-440. doi: $10.1159 / 000496210$

Llodra, J. C., Rodriguez, A., Ferrer, B., Menardia, V., Ramos, T., \& Morato, M. (2005). Efficacy of silver diamine fluoride for caries reduction in primary teeth and first permanent molars of schoolchildren: 36-month clinical trial. J Dent Res, 84(8), 721-724. doi:10.1177/154405910508400807

Lobo, M. M., Goncalves, R. B., Ambrosano, G. M., \& Pimenta, L. A. (2005). Chemical or microbiological models of secondary caries development around different dental restorative materials. J Biomed Mater Res B Appl Biomater, 74(2), 725-731. doi:10.1002/jbm.b.30253

Mei, M. L., Lo, E. C. M., \& Chu, C. H. (2018). Arresting Dentine Caries with Silver Diamine Fluoride: What's Behind It? J Dent Res, 97(7), 751-758. doi:10.1177/0022034518774783

Mei, M. L., Zhao, I. S., Ito, L., Lo, E. C., \& Chu, C. H. (2016). Prevention of secondary caries by silver diamine fluoride. Int Dent J, 66(2), 71-77. doi:10.1111/idj.12207

Roberts, A., Bradley, J., Merkley, S., Pachal, T., Gopal, J., \& Sharma, D. Does potassium iodide application following silver diamine fluoride reduce staining of tooth? A systematic review. $n / a(\mathrm{n} / \mathrm{a})$. doi:10.1111/adj.12743

Rosenblatt, A., Stamford, T. C., \& Niederman, R. (2009). Silver diamine fluoride: a caries "silver-fluoride bullet". J Dent Res, 88(2), 116-125. doi:10.1177/0022034508329406

Seifo, N., Robertson, M., MacLean, J., Blain, K., Grosse, S., Milne, R., . . Innes, N. (2020). The use of silver diamine fluoride (SDF) in dental practice. British Dental Journal, 228(2), 75-81. doi:10.1038/s41415-020-1203-9

Selwitz, R. H., Ismail, A. I., \& Pitts, N. B. (2007). Dental caries. Lancet, 369(9555), 51-59. doi:10.1016/S0140-6736(07)60031-2

Sorkhdini, P., Gregory, R. L., Crystal, Y. O., Tang, Q., \& Lippert, F. (2020). Effectiveness of in vitro primary coronal caries prevention with silver diamine fluoride - Chemical vs biofilm models. J Dent, 99, 103418. doi:https://doi.org/10.1016/j.jdent.2020.103418

Timms, L., Sumner, O., Deery, C., \& Rogers, H. J. (2020). Everyone else is using it, so why isn't the UK? Silver diamine fluoride for children and young people. Community Dent Health. doi:10.1922/CDH_00008Timms07

Vasquez, E., Zegarra, G., Chirinos, E., Castillo, J. L., Taves, D. R., Watson, G. E., ... Milgrom, P. (2012). Short term serum pharmacokinetics of diammine silver fluoride after oral application. BMC Oral Health, 12, 60. doi:10.1186/1472-6831-12-60 
Zhao, I. S., Chu, S., Yu, O. Y., Mei, M. L., Chu, C. H., \& Lo, E. C. M. (2019). Effect of silver diamine fluoride and potassium iodide on shear bond strength of glass ionomer cements to caries-affected dentine. Int Dent J, 69(5), 341-347. doi:10.1111/idj.12478

Zhao, I. S., Mei, M. L., Burrow, M. F., Lo, E. C., \& Chu, C. H. (2017a). Effect of Silver Diamine Fluoride and Potassium Iodide Treatment on Secondary Caries Prevention and Tooth Discolouration in Cervical Glass Ionomer Cement Restoration. Int J Mol Sci, 18(2). doi:10.3390/ijms 18020340

Zhao, I. S., Mei, M. L., Burrow, M. F., Lo, E. C., \& Chu, C. H. (2017b). Prevention of secondary caries using silver diamine fluoride treatment and casein phosphopeptide-amorphous calcium phosphate modified glass-ionomer cement. J Dent, 57, 38-44. doi:10.1016/j.jdent.2016.12.001

Zhao, I. S., Mei, M. L., Li, Q. L., Lo, E. C. M., \& Chu, C. H. (2017). Arresting simulated dentine caries with adjunctive application of silver nitrate solution and sodium fluoride varnish: an in vitro study. Int Dent J, 67(4), 206-214. doi:10.1111/idj.12291

Zhi, Q. H., Lo, E. C., \& Kwok, A. C. (2013). An in vitro study of silver and fluoride ions on remineralization of demineralized enamel and dentine. Aust Dent J, 58(1), 50-56. doi:10.1111/adj.12033

Zhi, Q. H., Lo, E. C., \& Lin, H. C. (2012). Randomized clinical trial on effectiveness of silver diamine fluoride and glass ionomer in arresting dentine caries in preschool children. $J$ Dent, 40(11), 962-967. doi:10.1016/j.jdent.2012.08.002

\section{Figure legends}

Fig. 1. Schematic of the experimental procedures.

Fig. 2. \%SMHchange data (mean \pm standard deviation) as a function of intervention after $\mathrm{pH}$ cycling with twice-daily fluoride (black bars) or placebo (gray bars) treatments. Different letters highlight statistically significant differences between treatment groups and within each model. Asterisks highlight statistically significant differences within treatment groups between models.

Fig. 3. Integrated mineral loss data ( $\Delta \mathrm{Z}$; mean \pm standard deviation) as a function of intervention after $\mathrm{pH}$ cycling with twice-daily fluoride (black bars) or placebo (gray bars) treatments. Different letters highlight statistically significant differences between treatment groups and within each model. Asterisks highlight statistically significant differences within treatment groups between models. 
Fig. 4. Lesion Depth (L; mean \pm standard deviation) data as a function of intervention after $\mathrm{pH}$ cycling with twice-daily fluoride (black bars) or placebo (gray bars) treatments. Different letters highlight statistically significant differences between treatment groups.

Fig. 5. Color change ( $\Delta \mathrm{L}^{*}$; mean \pm standard deviation) data for both models and all treatment groups. Different letters highlight statistically significant differences between treatment groups and within each model. Asterisks highlight statistically significant differences within treatment groups between models.

Fig. 6. Color changes between treatment groups 


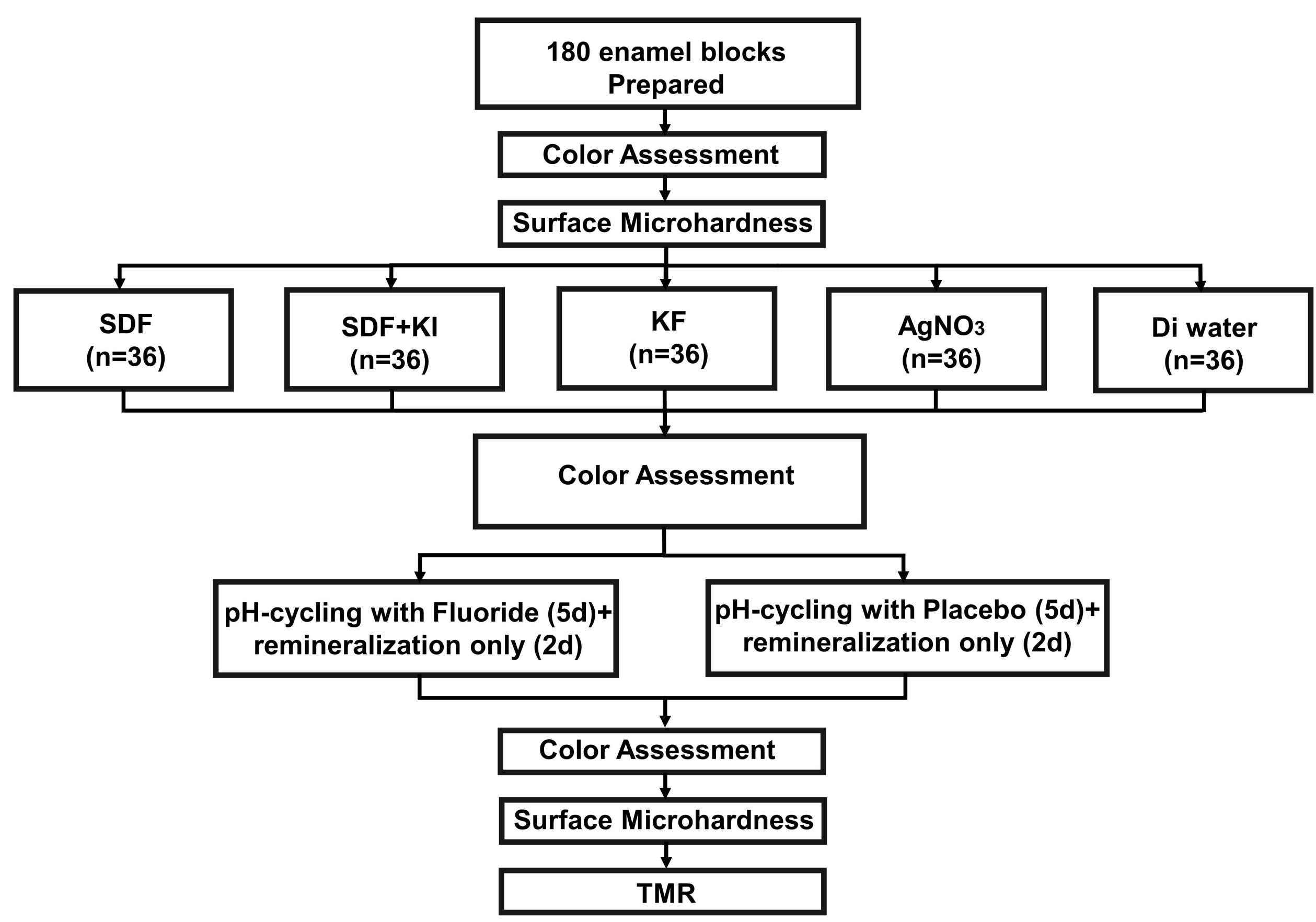




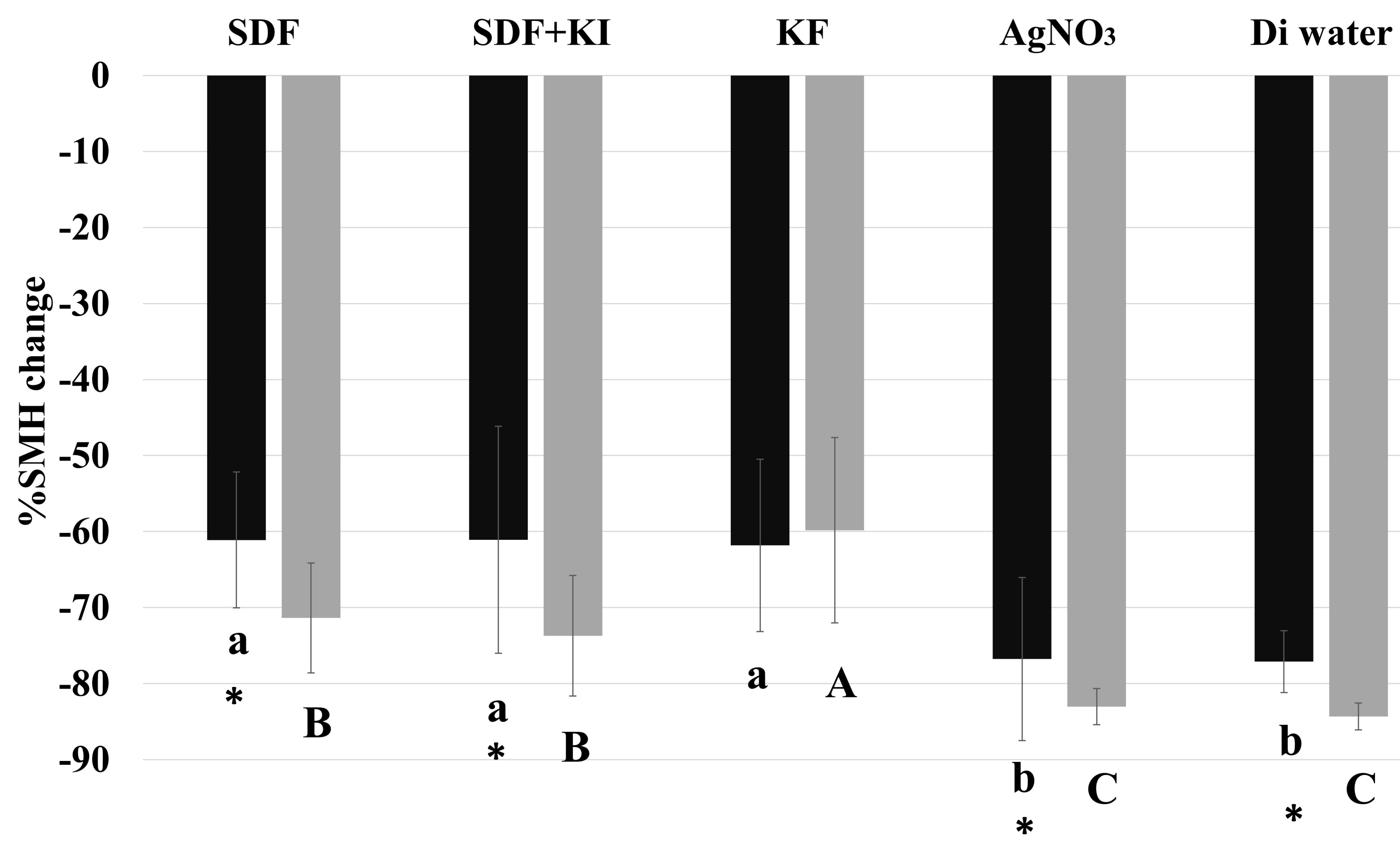


1400

B

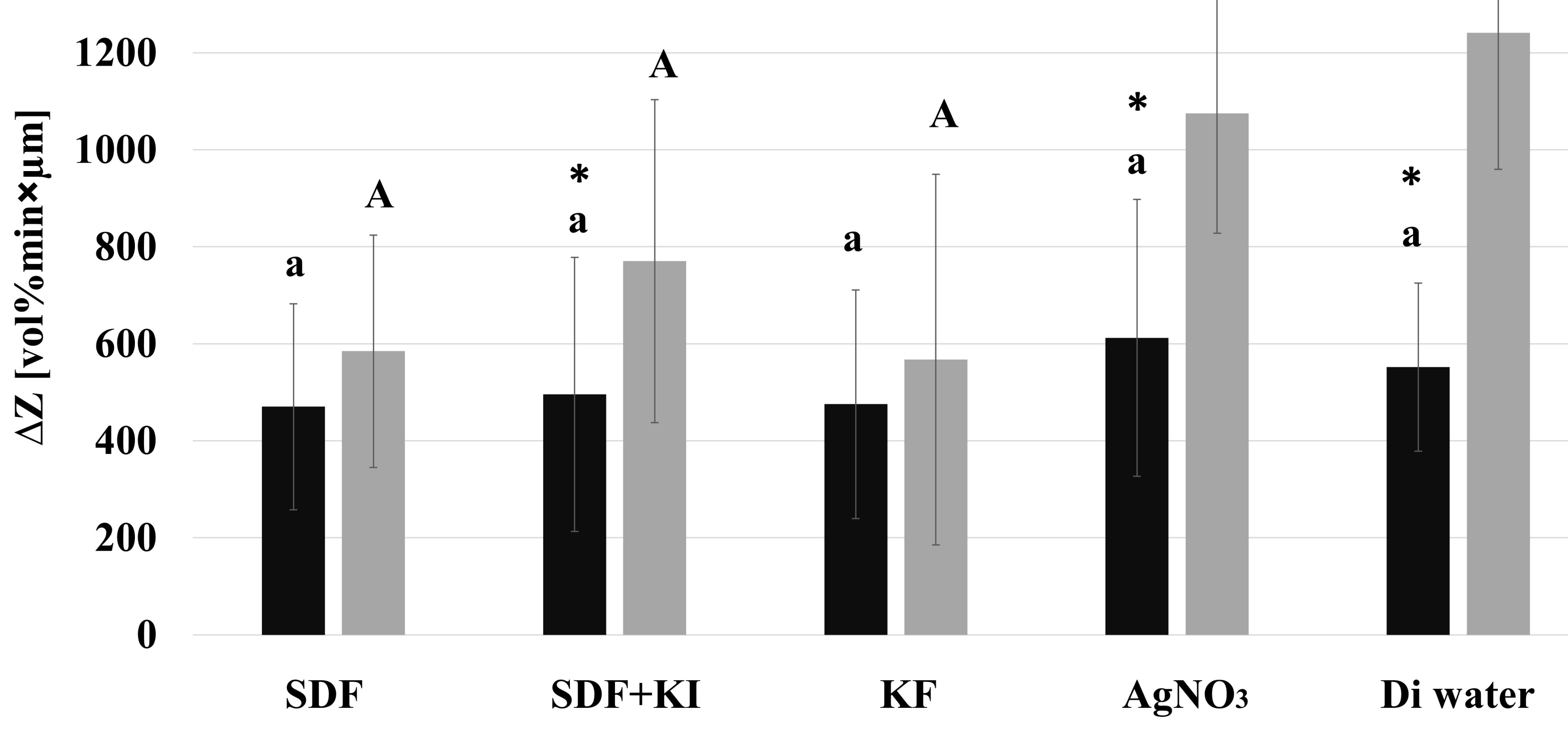


50

45

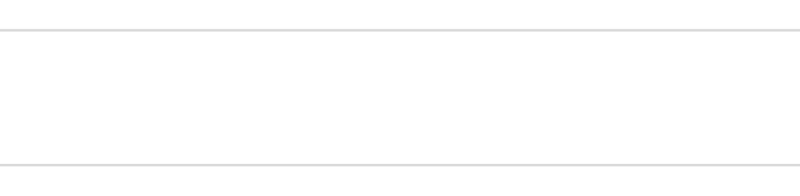

b

b

40

a

a

$\underbrace{35}_{25} \begin{aligned} & 35 \\ & 30\end{aligned}$

20

15

10

5

0
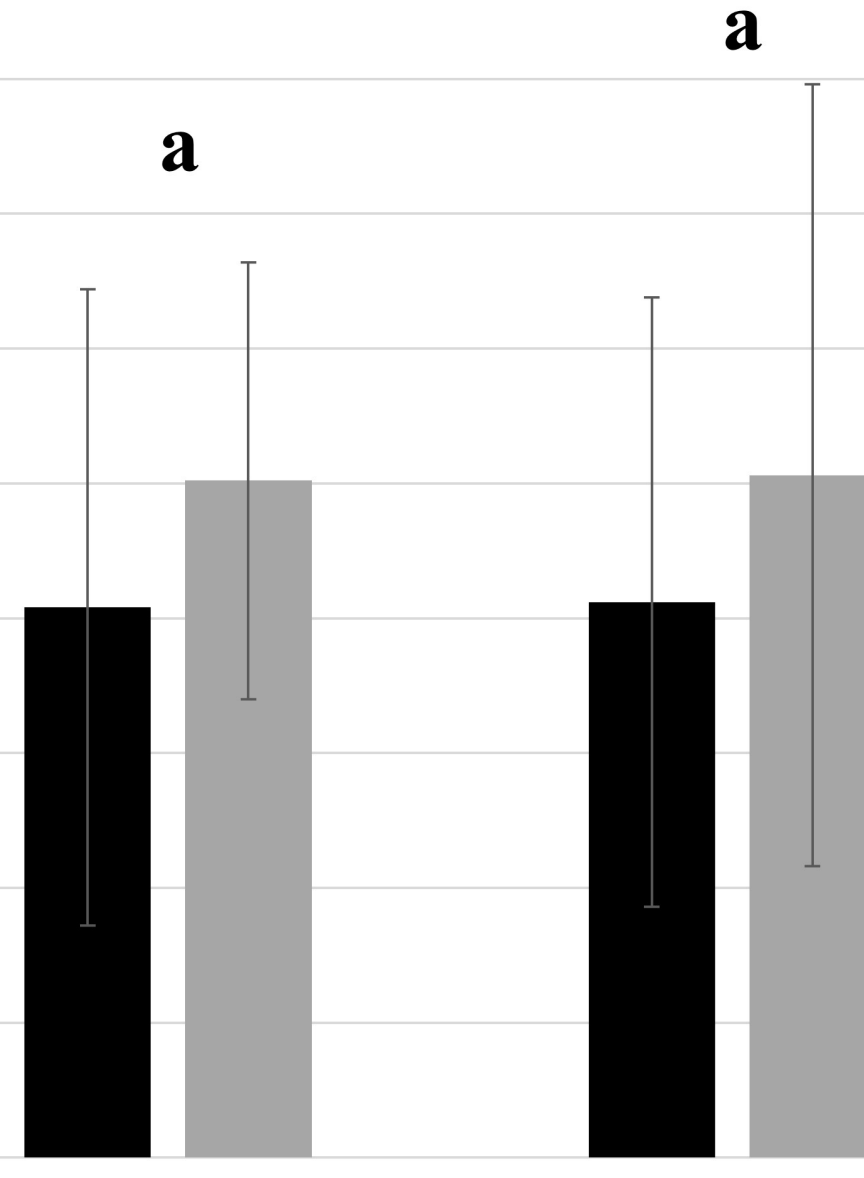

SDF+KI

KF

$\mathrm{AgNO}_{3}$

Di water 


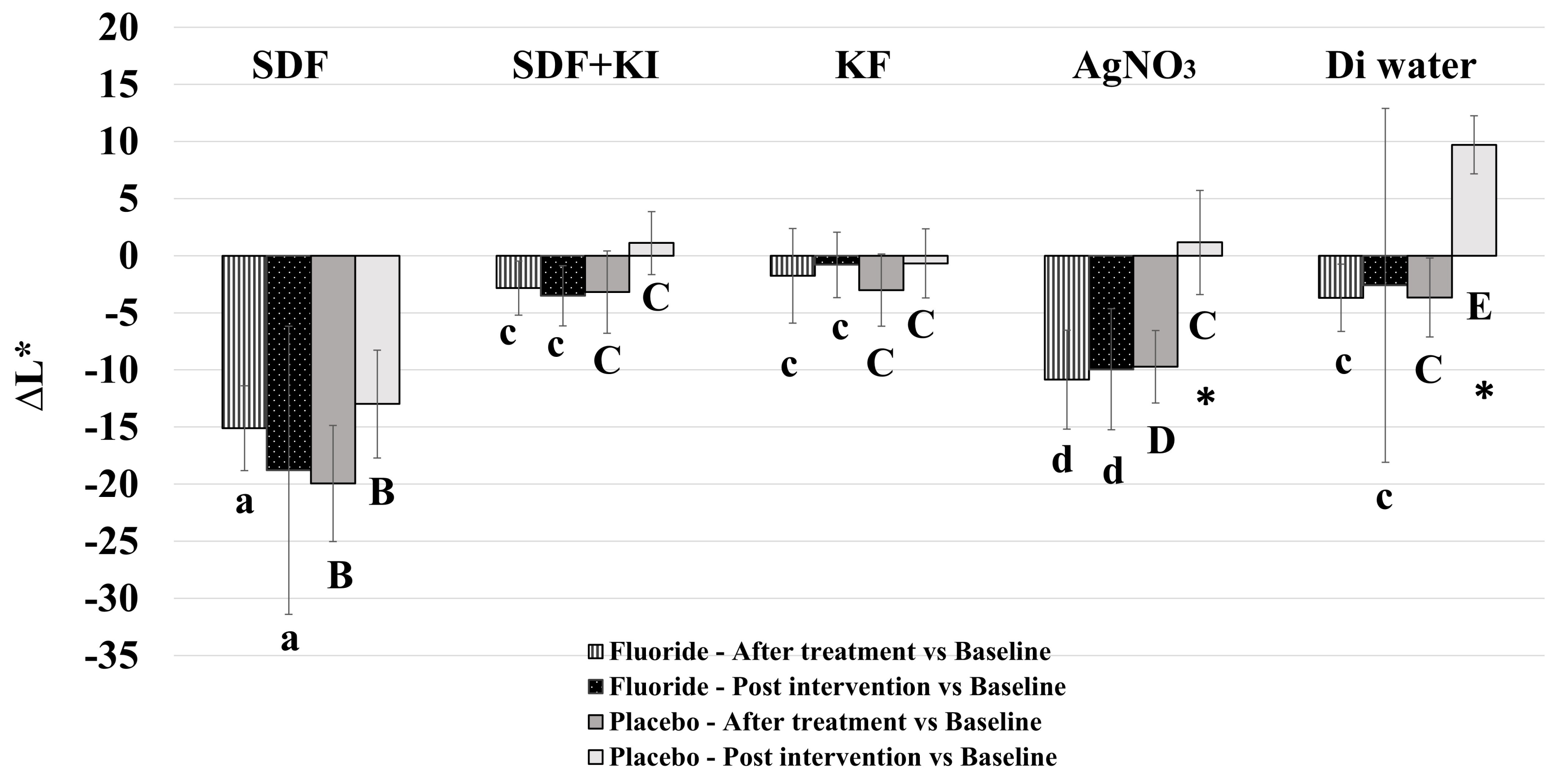


